\title{
An approximate consumption function
}

\author{
Mario Padula* \\ Very Preliminary and Very Incomplete
}

8 December 2005

\begin{abstract}
This notes proposes an approximation to the consumption function in the buffer-stock model.

Acknowledgement 1 I would like to than Chris Carroll, Alessandro De Martino, Tullio Jappelli and Luigi Pistaferri for comments and suggestions. The usual disclaimer applies.
\end{abstract}

\section{Introduction}

This paper provides a class of $C^{\infty}$ function to approximate the consumption function in the buffer stock model of saving. The approximation is derived for the Carroll's (1992) incarnation of the buffer stock model, but equally applies to the Deaton's (1991) version of such model. It relies on the monotonicity of the consumption function, on concavity, and on the fact that the consumption function is bounded from above and from below and so is its derivative.

The paper is organized as follows. Notation is lied down in Section 2. Section 3 reviews two class methods for numerically solving the model: a class of standard methods and the endogenous gridpoints algorithm. The approximation is derived and discussed in Section 4. Section 5 deals with two examples, one for a US parametrized economy, the other for Italy, while section 6 concludes.

\section{The notation}

Consumers live from time 0 to time $T$. They maximize:

$$
E_{0} \sum_{t=0}^{T} \beta^{t} u\left(C_{t}\right)
$$

${ }^{*}$ University of Salerno and CSEF. 
with respect to consumption, $C_{t}$, under the dynamic budget constraint:

$$
W_{t+1}=R\left[W_{t}+Y_{t}-C_{t}\right]
$$

where $\beta$ is the subjective discount factor, $W_{t+1}$ and $W_{t}$ are, respectively, nonhuman wealth at time $t+1$ and at time $t, R$ the interest factor and $Y_{t}$ labor income at time $t$. The utility function is assumed to be of the CRRA type, i.e.:

$$
u\left(C_{t}\right)=\frac{C_{t}^{1-\rho}}{1-\rho}
$$

where $\rho$ is the coefficient of relative risk aversion. Labor income shifts due to transitory and permanent shocks:

$$
\begin{gathered}
Y_{t}=P_{t} \Xi_{t} \\
P_{t}=G P_{t-1} \Psi_{t}
\end{gathered}
$$

where $P_{t}$ is permanent income, $\Xi_{t}$ is the transitory and $\Psi_{t}$ the permanent income shocks, $G$ is the growth factor of permanent income. Income is zero with a small probability $p$, i.e.:

$$
\Xi_{t+n}=\left\{\begin{array}{ccc}
0 & \text { with probability } & p>0 \\
\frac{\Theta_{t+n}}{q} & \text { with probability } & q \equiv 1-p
\end{array}\right.
$$

Furthermore, following Carroll (1992) we assume that transitory and permanent shocks are dawn from a log-normal distribution and that: $E_{t}\left[\Theta_{t+n}\right]=1$ for $n>$ 0 , that $\operatorname{var}\left(\log \Theta_{t+n}\right)=\sigma_{\theta}^{2}$, that $E_{t}\left[\Psi_{t+n}\right]=1$ and that $\operatorname{var}\left(\log \Psi_{t+n}\right)=\sigma_{\psi}^{2}$. Finally, it is assumed that consumer cannot die in debt, i.e.

$$
C_{T} \leq W_{T}+Y_{T}
$$

This last assumption naturally leads to use the dynamic programming principle. ${ }^{1}$ The Bellman equation for the consumer problem is:

$$
\begin{aligned}
& V_{t}\left(W_{t}, P_{t}\right)=\max _{C_{t}}\left\{u\left(C_{t}\right)+\beta E_{t} V_{t+1}\left(W_{t+1}, P_{t+1}\right)\right\} \\
& \text { s.t. } \\
& P_{t+1}=G P_{t} \Psi_{t+1} \\
& W_{t+1}=R\left[W_{t}-C_{t}+Y_{t}\right]
\end{aligned}
$$

In order to exploit the homogeneity of the utility function, one can define cashon-hand as:

$$
M_{t}=W_{t}+Y_{t}
$$

This allows to rewrite the Bellman equation as:

$$
\begin{aligned}
& v_{t}\left(m_{t}\right)=\max _{c_{t}}\left\{u\left(c_{t}\right)+\beta E_{t} G^{1-\rho} \Psi_{t+1}^{1-\rho} v_{t+1}\left(m_{t+1}\right)\right\} \\
& \text { s.t. } \\
& m_{t+1}=\frac{R}{G \Psi_{t+1}}\left[m_{t}-c_{t}\right]+\Xi_{t+1}
\end{aligned}
$$

\footnotetext{
${ }^{1}$ The non-stationary nature of the problem is not an issue in this context, thanks to the homogeneity of the objective function.
} 
where $m_{t}=M_{t} / P_{t}$ and $c_{t}=C_{t} / P_{t}$. Carroll (2004) shows that (1) defines a contraction mapping under three restrictions: (i) $G<R$; (ii) $(R \beta)^{\frac{1}{\rho}}<R$; (iii) $R \beta E_{t}\left[G \Psi_{t+n}^{-\rho}\right]<1$. The first condition guarantees that human capital does not explode; the second that consumers are not too patient; the third that consumers are impatient enough for cash-on-hand not to go to infinity. Carroll (2004) also shows that the consumption function is increasing, concave and that it is bounded from above and from below; moreover, that there exists a unique and stable level of cash-on-hand, the target $m^{*}$, such that $E_{t} m_{t+1}=m_{t}$ if $m_{t}=m^{*}$.

\section{Standard solution methods and the endoge- nous grid-point algorithm}

Problem (1) has not a closed form solution. This means that its solution requires employing numerical methods. The problem is naturally characterized as a recursive one, which means that the solution can be found by value or policy function iteration or using projection methods. ${ }^{2}$

A common solution strategy amounts to iterate Euler Equation for consumption, starting from $c_{T}=m_{T}$ :

$$
u^{\prime}\left(c_{t}\right)=\beta R E_{t}\left\{G^{-\rho} \Psi_{t+1}^{-\rho} v_{t+1}^{\prime}\left[\frac{R}{G \Psi_{t+1}}\left(m_{t}-c_{t}\right)+\Xi_{t+1}\right]\right\}
$$

where, from the envelope condition, $v_{t}^{\prime}\left(m_{t}\right)=u^{\prime}\left(c_{t}\right)$. In order to iterate the Euler equation, one needs to discretize the state-space. This amounts to define a grid for $m_{t}$, i.e. $\left\{\mu_{1}, \mu_{2}, \cdots, \mu_{I}\right\}$, to discretize the distribution of permanent and transitory income shocks. and solve:

$$
\begin{gathered}
u^{\prime}\left(\chi_{1}\right)=\beta R E_{t}\left\{G^{-\rho} \Psi_{t+1}^{-\rho} v_{t+1}^{\prime}\left[\frac{R}{G \Psi_{t+1}}\left(\mu_{1}-\chi_{1}\right)+\Xi_{t+1}\right]\right\} \\
u^{\prime}\left(\chi_{2}\right)=\beta R E_{t}\left\{G^{-\rho} \Psi_{t+1}^{-\rho} v_{t+1}^{\prime}\left[\frac{R}{G \Psi_{t+1}}\left(\mu_{2}-\chi_{2}\right)+\Xi_{t+1}\right]\right\} \\
\vdots \\
\vdots \\
u^{\prime}\left(\chi_{I}\right)=\beta R E_{t}\left\{G^{-\rho} \Psi_{t+1}^{-\rho} v_{t+1}^{\prime}\left[\frac{R}{G \Psi_{t+1}}\left(\mu_{I}-\chi_{I}\right)+\Xi_{t+1}\right]\right\}
\end{gathered}
$$

with respect to $\left\{\chi_{1}, \chi_{2}, \cdots, \chi_{I}\right\}$. The consumption function is then obtained by interpolating the couples $\left\{\left(\chi_{1}, \mu_{1}\right),\left(\chi_{2}, \mu_{2}\right), \cdots,\left(\chi_{I}, \mu_{I}\right)\right\}$. Solving system (2) requires evaluating the expected value of the marginal utility of consumption at each of the grid points. This entails a substantial amount of computer time, for fine enough state-space grids.

\footnotetext{
${ }^{2}$ For an introductory treatment of the topic see Adda and Cooper, 2003; more advanced readers might want to look at Judd, 1998
} 
The endogenous gridpoints algorithm improves on standard solution methods (see Carroll, 2005). Instead of defining a grid for $m_{t}$, the algorithm requires discretizing $m_{t}-c_{t}$, i.e. the end of period asset, and solving:

$$
\begin{gathered}
\chi_{1}=u^{\prime}-1\left\{\beta R E_{t}\left[G^{-\rho} \Psi_{t+1}^{-\rho} v_{t+1}^{\prime}\left(\frac{R}{G \Psi_{t+1}} \alpha_{1}+\Xi_{t+1}\right)\right]\right\} \\
\chi_{2}=u^{\prime}-1\left\{\beta R E_{t}\left[G^{-\rho} \Psi_{t+1}^{-\rho} v_{t+1}^{\prime}\left(\frac{R}{G \Psi_{t+1}} \alpha_{2}+\Xi_{t+1}\right)\right]\right\} \\
\vdots \\
\vdots \\
\chi_{I}=u^{\prime}-1\left\{\beta R E_{t}\left[G^{-\rho} \Psi_{t+1}^{-\rho} v_{t+1}^{\prime}\left(\frac{R}{G \Psi_{t+1}} \alpha_{I}+\Xi_{t+1}\right)\right]\right\}
\end{gathered}
$$

where $\left\{\alpha_{1}, \alpha_{2}, \cdots, \alpha_{I}\right\}$ is the grid for the end of period asset. The endogenous gridpoints algorithm is more efficient than other standard methods since it evaluates expectations only for points used in the interpolating functions. This translates into non-negligible savings in the amount of computer time needed to solve the consumers problem. We thus compare our approximate consumption function with that obtained using the endogenous grid-point method.

\section{The approximate consumption function}

Carroll (2004) shows that the consumption function, $c(m)$, satisfies the following properties:

- $c(m)$ is a $C^{\infty}$ function, from $\mathcal{R}^{+}$to $\mathcal{R}^{+}$.

- $c(0)=0$ and $\lim _{m \rightarrow \infty} c(m)=\infty$.

- $c(m)$ is strictly increasing and concave, i.e $c^{\prime}(m)>0$ and $c^{\prime \prime}(m)<0$.

- $\lim _{m \rightarrow 0} c^{\prime}(m)=\bar{\kappa}$ and $\lim _{m \rightarrow \infty} c^{\prime}(m)=\underline{\kappa}$ with $\bar{\kappa}>\underline{\kappa}>0$ and $\bar{\kappa}, \underline{\kappa} \in \mathcal{R}^{+}$.

Our approximate consumption function is given by:

$$
c(m)=2(\bar{\kappa}-\underline{\kappa})\left[m-\frac{1}{b}(\log (1+\exp (b m)-\log (2))]+\underline{\kappa} m\right.
$$

where:

$$
\begin{aligned}
& \bar{\kappa}=1-R^{-1}(R \beta p)^{\frac{1}{\rho}} \\
& \underline{\kappa}=1-R^{-1}(R \beta)^{\frac{1}{\rho}}
\end{aligned}
$$

where $b$ is a strictly positive real. Differentiating (4) one obtains the Fermi-Dirac distribution, i.e.:

$$
c^{\prime}(m)=\frac{2(\bar{\kappa}-\underline{\kappa})}{1+\exp (b m)}+\underline{\kappa}
$$


The parameter $b$ governs the degree of concavity of the consumption function. The higher $b$, the sooner the derivative of approximate consumption function converges to its limit, $\underline{\kappa}$. Carroll and Kimball (1996) show that uncertainty reduces consumption for each level of cash-on-hand. So the higher is uncertainty, the larger has to be $b$. Figure 1 plots the approximate consumption function for various values of $b$ and for $G=1.015, R=1.025, \rho=2, \beta=0.96$, and $p=0.005$.

Given the growth factor of income, the interest factor, the probability of unemployment, the discount factor and the relative risk aversion, $b$ is set to make the approximate consumption function as much close to the true consumption function. The next section compares the approximate consumption with the consumption function obtained employing the endogenous grid point algorithm to solve the consumers problem in a US and an Italy parametrized economy. Since in the two economy agents face a very diverse degree of uncertainty, the exercise will help to understand how the quality of the approximation varies with uncertainty.

\section{Two examples}

\section{To be written.}

\section{Conclusions}

To be written. 


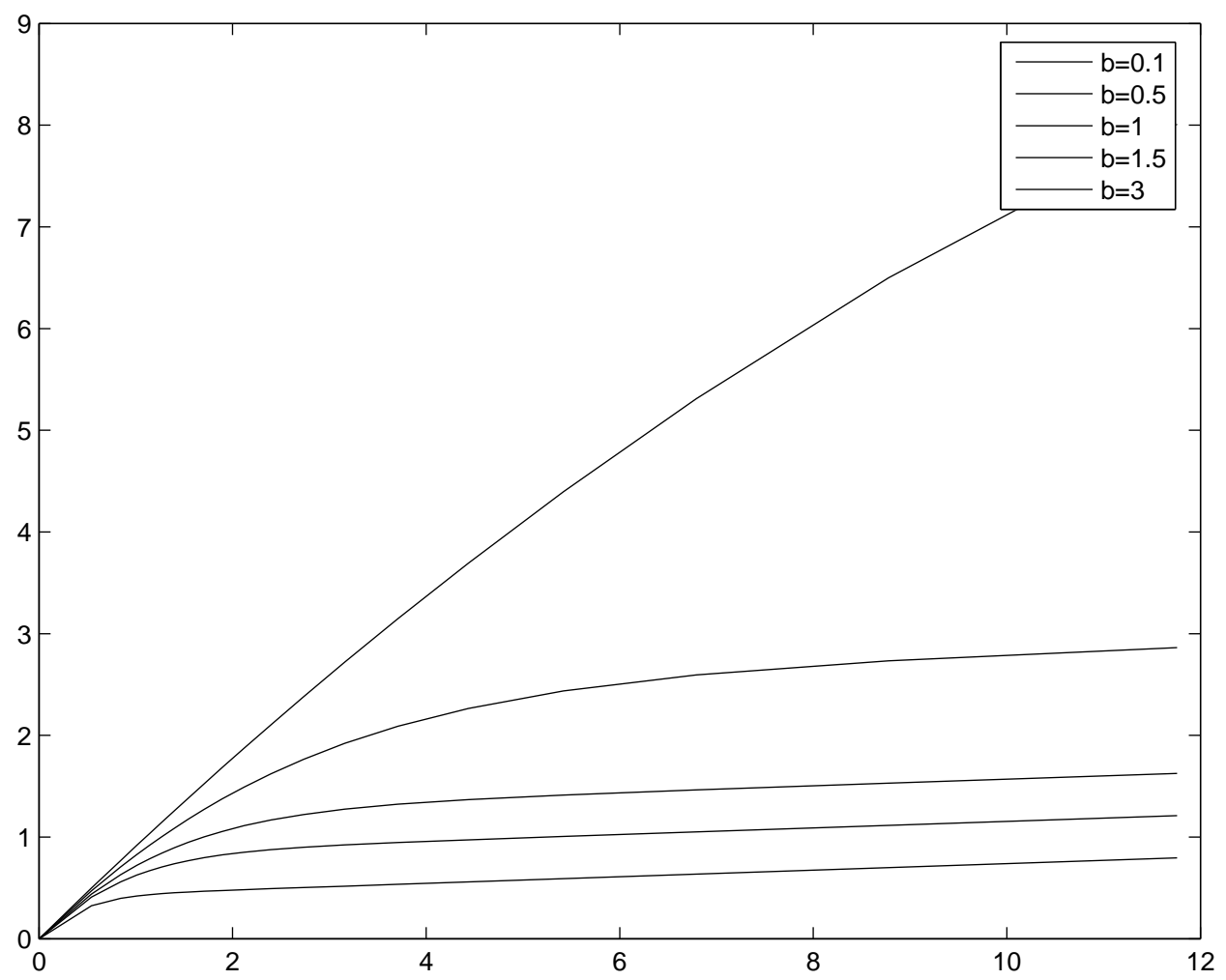

Figure 1: The approximate consumption function

\section{References}

[1] Adda, Jérôme and Russel Cooper (2003), "Dynamic Economics: Quantitative Methods and Applications," MIT Press.

[2] Carroll, Christopher D. (1992), "The Buffer-Stock Theory of Saving: Some Macroeconomic Evidence," Brookings Papers on Economic Activity 1, 61156.

[3] Carroll, Christopher D. and Miles Kimball (1996), "On the Concavity of The Consumption Function," Econometrica, 64(4),981-992.

[4] Carroll, Christopher D. (1997), "Buffer-Stock Saving and the Life Cycle/Permanent Income Hypothesis," Quarterly Journal of Economics 112, 1-56. 
[5] Carroll, Christopher D. (2001), "A Theory of the Consumption Function, with and without Liquidity Constraints," Journal of Economic Perspectives 15, 23-45.

[6] Carroll, Christopher D. (2004), "Theoretical Foundations of Buffer Stock Saving," John Hopkins University, Working Paper n. 517.

[7] Carroll, Christopher D., and Andrew A. Samwick (1997), "The Nature of Precautionary Wealth," Journal of Monetary Economics 40, 41-71.

[8] Carroll, Christopher D. (2005), "The Method of Endogenous Gridpoints for Solving Dynamic Stochastic Optimization Problems," downloadable at http://econ.jhu.edu/people/ccarroll/EndogenousArchive.zip

[9] Deaton, Angus S. (1991), "Saving and Liquidity Constraints," Econometrica 59, 1221-48.

[10] Judd, Kenneth L. (1998), "Numerical Methods in Economics," MIT Press. 